\title{
Influence of Anesthesia Type on Outcomes after Endovascular Treatment in Acute Ischemic Stroke: Meta-Analysis
}

\author{
Chulho Kim, $\mathrm{MD}, \mathrm{PhD}^{1}$, Sung-Eun Kim, $\mathrm{MD}^{2}$, Jin Pyeong Jeon, $\mathrm{MD}, \mathrm{PhD}^{3,4,5}$ \\ ${ }^{1}$ Department of Neurology, Hallym University College of Medicine, Chuncheon, Korea \\ ${ }^{2}$ Department of Emergency Medicine, Seoul Emergency Operations Center, Seoul, Korea \\ ${ }^{3}$ Department of Neurosurgery, Hallym University College of Medicine, Chuncheon, Korea \\ ${ }^{4}$ Institute of New Frontier Stroke Research, Hallym University College of Medicine, Chuncheon, Korea \\ ${ }^{5}$ Genetic and Research, Hallym University College of Medicine, Chuncheon, Korea
}

Purpose: To assess clinical and angiographic outcomes after endovascular treatment (EVT) in ischemic stroke patients according to anesthesia types (general anesthesia vs. conscious sedation).

Materials and Methods: A systematic literature review through an online data base between January 1990 and September 2017 was performed. A fixed effect model was used in cases of $<50 \%$ heterogeneity. The primary outcomes were good clinical outcome at the 3-month follow-up and successful recanalization. A meta-regression analysis was done to estimate primary outcomes of log odds ratio (OR) on onset-to-puncture time (OTP) differences. Publication bias was determined using Begg's funnel plot and additional the Trim and Fill method.

Results: Sixteen articles including 2,662 patients (general anesthesia, $n=1,275$; conscious sedation, $n=1,387$ ) were included. General anesthesia significantly decreased good outcomes than conscious sedation (OR, 0.564; 95\% confidence interval [Cl], 0.354-0.899). However, outcomes did not differ significantly in randomized controlled trials (RCTs; OR, 1.101; 95\% Cl, 0.395-3.071). Anesthesia type was not associated with successful recanalization (OR, 0.985; 95\% $\mathrm{Cl}, 0.787-1.233)$. General anesthesia increased the risk of mortality $(\mathrm{OR}, 1.532 ; 95 \% \mathrm{Cl}, 1.187-1.976)$ and pneumonia (OR, 1.613; 95\% Cl, 1.172-2.221), but not symptomatic intracranial hemorrhage $(\mathrm{OR}, 1.125 ; 95 \% \mathrm{Cl}, 0.767-1.652)$. The meta-regression analysis showed no linear relationship between OTP differences and log OR of good outcome (coefficient, $0.0004 ; \mathrm{P}=0.95$ ) or successful recanalization (coefficient, $0.0005 ; \mathrm{P}=0.94$ ), respectively.

Conclusion: General anesthesia seemed to be associated with adverse clinical outcome after EVT. However, its efficacy was not demonstrated in RCTs. Successful recanalization did not differ according to anesthesia type. Studies using individual patient data based on further RCTs are necessary to elucidate anesthesia effect on procedural and clinical outcomes.

Key Words: Anesthesia, General; Stroke 


\section{INTRUDOCTION}

Endovascular treatment (EVT) has shown better outcomes than intravenous thrombolysis alone in patients with acute ischemic stroke of anterior circulation.' Accordingly, avoiding procedure-related complications is the main concern. Successful EVT requires immobilization of the patients due to construction of angiographic roadmap for microcatheter navigation. However, in clinical circumstances, poor patient cooperation due to agitation or altered level of conscious frequently can occur during procedures involving general anesthesia (GA) with intubation and conscious sedation (CS) without intubation. The optimal anesthesia for EVT remains unsolved. Advantages of GA include breathing control and airway maintenance without patient movement. Advantages of CS include better hemodynamic stability, less time-consuming task of initiating groin puncture and ability to assess patients' neurologic status during and after EVT. ${ }^{2}$

Retrospective investigations suggested that GA was associated with adverse outcomes. Sugg et al. ${ }^{3}$ reported that good outcome was significantly decreased in patients with GA (11.1\% vs. 50.9\% in CS; P=0.033). Abou-Chebl et al. ${ }^{4}$ also demonstrated that GA significantly increased poor clinical outcome (odds ratio [OR], 2.4; $\mathrm{P}=0.01)$ and mortality (OR, 3.3; $\mathrm{P}=0.001$ ). However, difference in higher baseline NIH Stroke Scale (NIHSS) score ${ }^{4}$ and older age ${ }^{3}$ in GA patients can be concern to interpretation. Two recent randomized controlled trials $(\mathrm{RCTs})^{2}$ revealed no association between anesthesia type and clinical outcomes. Löwhagen Hendén et al. ${ }^{2}$ reported no differences in early neurologic improvement (NIHSS score shift within 24 hours after intervention, 9 [4-17] in GA vs. 8 [2.5-13] in $C S ; P=0.272$ ) and good outcomes at 3 months ( $n=19,42.2 \%$ in $G A$ vs. $18,40.0 \%$ in $C S ; P=1.00$ ). Schönenberger et al. ${ }^{5}$ also reported no neurologic improvement according to anesthesia type in acute anterior circulation stroke after thrombectomy. However, their data was derived from a relative small number of GA patients. A previous meta-analysis ${ }^{6}$ showed that ischemic stroke patients had undergone GA had lower good functional outcome (OR, 0.43; 95\% confidence interval [Cl], 0.35-0.53) and successful recanalization ( $\mathrm{OR}, 0.54 ; 95 \% \mathrm{Cl}, 0.37-0.80)$, but experienced more respiration complications (OR, 2.09; 95\% Cl, 1.36-3.23). Their conclusion was obtained from an electronic database searched until March 2014. Since 2014, studies comparing the two anesthetic methods have been increasingly reported. Accordingly, an updated meta-analysis is necessary to reveal anesthesia effect on EVT outcomes. ${ }^{6}$ This study compares treatment outcomes according to anesthesia types (GA vs. CS) during the procedures.

\section{MATERIALS AND METHODS}

\section{Literature search and selection criteria}

Electronic database through PubMed, EMBASE, and the Cochrane central database from January 1990 and May 2017 were searched using MeSH terms or key words. ${ }^{6,7}$ The search strategy is detailed in the supplemental data. The inclusion criteria for this meta-analysis were: 1) studies comparing treatment outcomes according to anesthesia types (GA vs. CS or local anesthesia; ${ }^{6}$ 2) age $>18$ years; 3 ) endovascular treatment including mechanical thrombectomy using stent retrieval, Merci retriever or suction devices or intra-arterial thrombolysis using tissue plasminogen activator or urokinase; and 4) capability of angiographic and clinical outcomes to be extracted. Risk of bias was assessed using the Cochrane risk of bias for RCTs (Supplementary Fig. 1) and Newcastle-Ottawa scale for non-randomized studies (Table 1). ${ }^{8}$ The exclusion criteria were: 1) lack of outcome separation according to anesthesia types; 2) overlapping data; 3) absence of interest outcomes; 4) no extractable data; 5) inclusion of only posterior circulation stroke; 6) review articles or case reports; 7) not officially approved articles; 8) EVT using ultrasound; ;,10 and 9) studies not reported in English."

\section{Outcome variables}

The primary outcomes were good clinical outcome at the 3-month follow-up and successful recanalization at the final angiogram. Secondary outcomes were 3-month mortality, symptomatic intracranial hemorrhage $(\mathrm{S}-\mathrm{ICH})$ and pneumonia. Good clinical outcome was defined as a 3-month modified Rankin scale score of $\leq 2$. Successful recanalization was defined as thrombolysis in cerebral ischemia $(\mathrm{TICl}) \geq 2 \mathrm{~b}$ or thrombolysis in Myocardial infarction grades $\geq 2 .{ }^{12} \mathrm{~S}-\mathrm{ICH}$ was defined as any $\mathrm{ICH}$ concomitant an increase of at least 4 baseline NIHSS score within 24 hours or death. ${ }^{13}$ Two authors (J.P.J. and C.K.) independently evaluated the eligibility of the studies and extracted the data using a uniform standardized form. Disagreements between two authors were resolved by discussion and consultation with a third author. This study was approved by the Institutional Review Boards of Chuncheon Sacred Heart Hospital. This meta-analysis was 


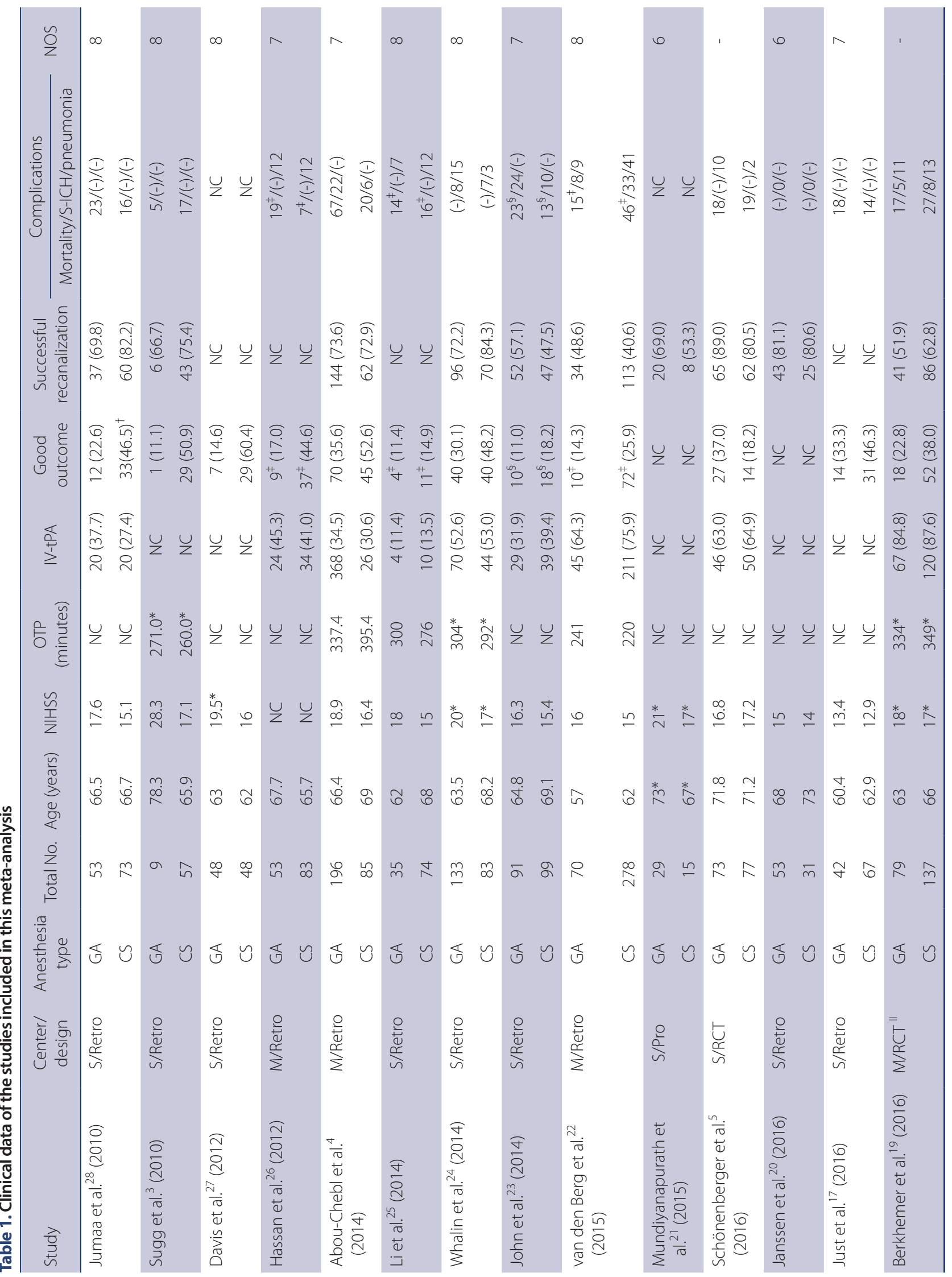




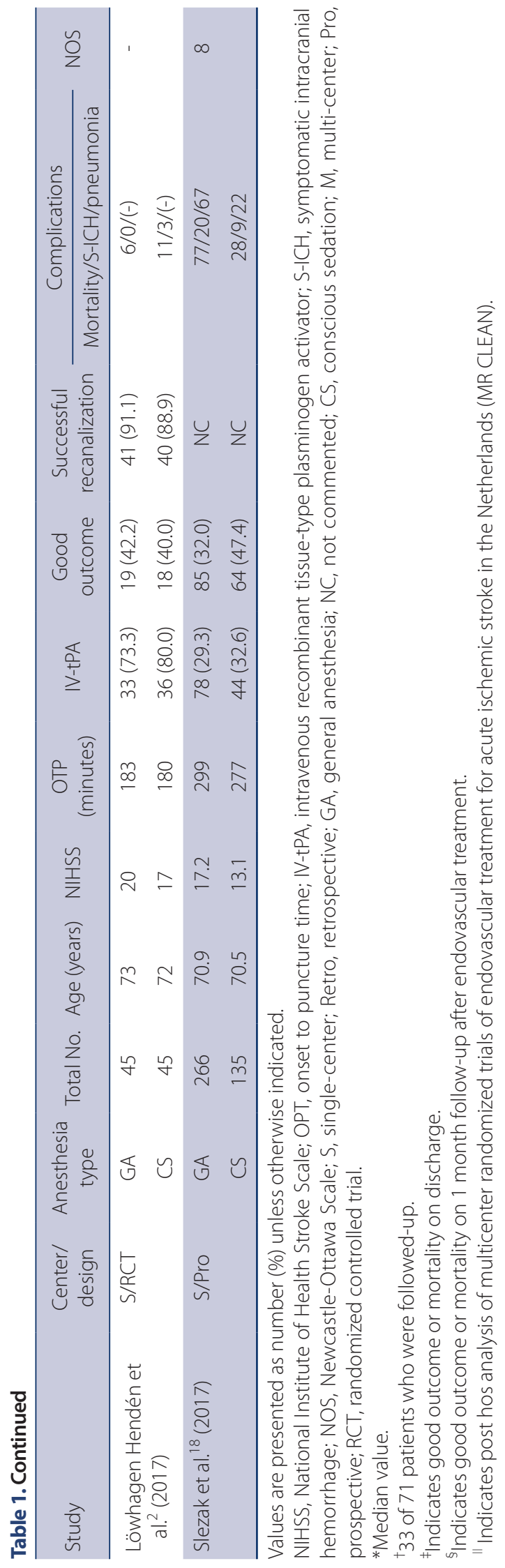

performed according to the PRISMA guidelines.

\section{Statistical analysis}

The cumulative incidence (event rate) and 95\% Cl were estimated from each study. Dichotomous variables are presented as OR with a $95 \% \mathrm{Cl}$. Heterogeneity was evaluated by using the $\mathrm{I}^{2}$ test. If $\mathrm{I}^{2}$ was $<50 \%$, a fixed effect model was used. Meta-regression analysis was performed to determine whether onset-to-puncture time (OTP) differences could affect primary outcomes according to anesthesia type. Publication bias was initially determined using Begg's funnel plot. The Trim-and-Fill method was performed further to estimate the number and outcome of missing studies. ${ }^{14-16}$ The comprehensive meta-analysis (CMA) software (CMA v2.2.064; Biostat, Englewood, NJ, USA) was used for all the above, with statistical significance defined as $\mathrm{P}<0.05$.

\section{RESULTS}

\section{Literature search}

A flow diagram of the detailed search process in this meta-analysis is provided in Fig. 1. After record screening and eligibility decisions, 27 articles were included. Among them, 11 were excluded in the final analysis due to no extractable data $(n=7)$, absence of interest outcomes ( $n=3)$ or study protocol ( $n=1)$ (Supplementary Table 1). Finally, 16 articles were included in the meta-analysis. ${ }^{2-5,17-28}$ Detailed information on the baseline characteristics are described in Table 1. The mean age for each study ranged between 60.4 and 78.3 years. The mean NIHSS score at admission for GA ranged from 13.4 to 28.3 and from 12.9 to 17.2 for CS.

\section{Outcomes after EVT}

Ten studies with 1,749 patients reported 3-month follow-up clinical outcomes. There were 367 (38.9\%) good clinical outcomes in GA and 372 (46.2\%) in CS. GA significantly decreased good functional outcomes compared to CS (OR, 0.564; 95\% Cl, 0.354-0.899; P=0.016; Fig. 2A). However, outcomes did not differ significantly according to anesthesia type (OR, 1.101; 95\% Cl, 0.395-3.071; $\mathrm{P}=0.854)$ in the $\mathrm{RCTs}$. 2,5,19 Regarding angiographic outcome, one study ${ }^{18}$ was excluded since it provided recanalization degree of $\mathrm{TICl} 2-3$. Anesthesia type was not associated with successful recanalization (OR, 0.985; 95\% Cl, 0.787-1.233; P=0.898; Fig. 2B). For three RCTs, successful recanalization did not differ significantly (OR, 1.067; 
$95 \% \mathrm{Cl}, 0.491-2.319 ; \mathrm{P}=0.869)$

GA increased the risk of morality $(\mathrm{OR}, 1.532 ; 95 \% \mathrm{Cl}, 1.187-$ 1.976; $P=0.001)$. However, the risk did not differ significantly in RCTs (OR, 0.923; 95\% Cl, 0.585-1.458; $\mathrm{P}=0.732)$. The occurrence of S-ICH did not differ significantly between two anesthesia types (OR, 1.125; 95\% Cl, 0.767-1.652; P=0.546). Pneumonia was more frequently observed in patients who underwent GA (OR, 1.613; 95\% Cl, 1.172-2.221; $P=0.003$ ) (Table 2). In two $\mathrm{RCTS},{ }^{5,19} \mathrm{GA}$ marginally increased the risk of pneumonia compared to CS (OR, 2.112; 95\% Cl, 0.998-4.469; $\mathrm{P}=0.051$ ) (data not shown).

\section{Meta-regression and publication bias}

A meta-regression analysis was done to estimate good clinical outcome or successful recanalization of log OR on OTP differences. The regression coefficient was $0.0004(P=0.95)$ in good outcome and $0.0005(P=0.94)$ in successful recanalization. The effect of OTP differences on the log OR of good outcome or successful recanalization was statistically insignificant. Accordingly, a linear relationship was not noted between OTP differences and log ORs (Fig. 3).

In the publication bias analysis for good outcome, funnel plot showed a relatively asymmetric appearance, indicating possible publication bias (Supplementary Fig. 2A). We trimmed two studies to resolve publication bias. The adjusted OR was 0.696 (95\% Cl, 0.424-0.886), suggesting no sig- nificant association between anesthesia type and 3-month outcome (Supplementary Table 2). The funnel plot dealing with successful recanalization showed symmetric pattern, suggesting absence of publication bias (Supplementary Fig. 2B).

\section{DISCUSSION}

The clinical efficacy of anesthesia type in outcomes after EVT remains inconclusive, although retrospective studies have shown adverse effects of GA. Presently, GA had lower good outcomes (OR, 0.564; 95\% Cl, 0.354-0.899) and higher mortality (OR, 1.532; 95\% Cl, 1.187-1.976), but possible publication bias is a limitation to the interpretation. In addition, such associations were not replicated in the RCTs (good outcomes: OR, 1.101; 95\% Cl, 0.395-3.071; mortality: OR, 0.923; 95\% Cl, 0.585-1.458; $P=0.732)$. No meaningful relationship by type of anesthesia was noted for successful recanalization (OR, 0.985; 95\% Cl, 0.787-1.233) and post-procedural S-ICH development (OR, 1.125; 95\% Cl, 0.767-1.652). GA significantly increased pneumonia (OR, 1.613; 95\% Cl, 1.172-2.221). The association was marginally significant for RCTs (OR, 2.112; 95\% Cl, $0.998-$ 4.469; $P=0.051)$.

Comparative studies of EVT outcomes according to anesthesia type are important, because most sedation choice are

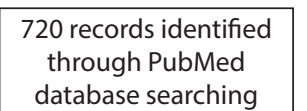
424 records identified through EMBASE database searching
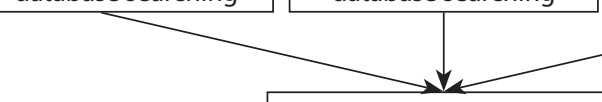

862 records identified after duplicates removed

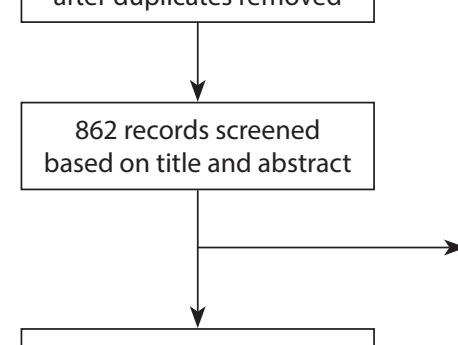

Excluded $(\mathrm{n}=835)$

Review or comment $(n=62)$

Case report or letter $(n=82)$

New technique $(n=31)$

Basic research $(n=62)$

Surgical treatment $(n=39)$

Irrelevant topics $(\mathrm{n}=559)$

Excluded $(n=11)$

Study protocal $(n=1)$

Absence of interest outcome $(n=3)$

No extractable data $(n=7)$

Fig. 1. Flow diagram for identification of relevant studies. 


\begin{tabular}{|c|c|c|c|c|c|c|c|}
\hline$\underline{\text { Study name }}$ & \multicolumn{2}{|c|}{ Events/total } & \multicolumn{5}{|c|}{$\underline{\text { Statistics for each study }}$} \\
\hline $\mathrm{RCT}$ & $\mathrm{GA}$ & CS & OR & $\begin{array}{c}\text { Lower } \\
\text { limit }\end{array}$ & $\begin{array}{c}\text { Upper } \\
\text { limit }\end{array}$ & Z-value & $P-v$ \\
\hline Schönenberger et al. ${ }^{5}$ (2016) & $27 / 73$ & $14 / 77$ & 2.641 & 1.249 & 5.587 & 2.541 & \\
\hline Berkhemer et al. ${ }^{19}$ (2016) & $18 / 79$ & $52 / 137$ & 0.482 & 0.257 & 0.905 & -2.272 & \\
\hline Löwhagen Hendén et al. $^{2}$ (2017) & $19 / 45$ & $18 / 45$ & 1.096 & 0.473 & 2.539 & 0.214 & \\
\hline & & & 1.101 & 0.395 & 3.071 & 0.184 & \\
\hline
\end{tabular}

Heterogeneity: $\mathrm{X}^{2}=11.667, \mathrm{df}=2(\mathrm{P}=0.003) ; \mathrm{I}^{2}=82.858 \%$

Statistics for each study

\begin{tabular}{|c|c|c|c|c|c|c|c|}
\hline Study name & Events & $\mathrm{s} /$ total & & Statisti & s for ea & h study & \\
\hline Non-RCT & GA & CS & OR & $\begin{array}{c}\text { Lower } \\
\text { limit }\end{array}$ & $\begin{array}{l}\text { Upper } \\
\text { limit }\end{array}$ & Z-value & P-value \\
\hline Jumaa et al. ${ }^{28}(2010)$ & $12 / 53$ & $33 / 71$ & 0.337 & 0.152 & 0.746 & -2.683 & 0.007 \\
\hline Sugg et al. ${ }^{3}(2010)$ & $1 / 9$ & $29 / 57$ & 0.121 & 0.014 & 1.029 & -1.934 & 0.053 \\
\hline Davis et al. ${ }^{27}$ (2012) & $7 / 48$ & $29 / 48$ & 0.112 & 0.042 & 0.301 & -4.343 & 0.000 \\
\hline Abou-Chebl et al. ${ }^{4}$ (2014) & $144 / 196$ & $62 / 85$ & 1.027 & 0.579 & 1.824 & 0.092 & 0.927 \\
\hline Whalin et al. ${ }^{24}$ (2014) & $40 / 133$ & $40 / 83$ & 0.462 & 0.262 & 0.816 & -2.661 & 0.008 \\
\hline Just et al. ${ }^{17}$ (2016) & $14 / 42$ & $31 / 67$ & 0.581 & 0.261 & 1.294 & -1.330 & 0.184 \\
\hline Slezak et al. ${ }^{18}$ (2017) & $85 / 226$ & $64 / 135$ & 0.521 & 0.341 & 0.797 & -3.008 & 0.003 \\
\hline & & & 0.432 & 0269 & 0.693 & -3.478 & 0.001 \\
\hline
\end{tabular}

Heterogeneity: $X^{2}=17.738, d f=6(P=0.007) ; I^{2}=66.174 \%$

OR and $95 \% \mathrm{Cl}$

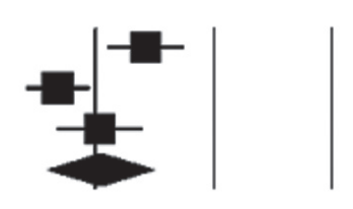

0.01

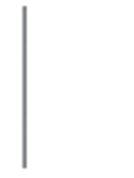

0.1 OR and $95 \% \mathrm{Cl}$

\begin{tabular}{llllll}
\hline Total & 0.564 & 0.354 & 0.899 & -2.411 & 0.116
\end{tabular}

Heterogeneity: $X^{2}=37.355, \mathrm{df}=9(\mathrm{P}=0.000) ; \mathrm{I}^{2}=75.907 \%$

Test for overall effect: $Z=-2.411(P=0.016)$

$$
\begin{array}{lllll}
0.564 & 0.354 & 0.899 & -2.411 & 0.116
\end{array}
$$

\begin{tabular}{|c|c|c|c|c|c|c|c|}
\hline Study name & \multicolumn{2}{|c|}{ Events/total } & \multicolumn{5}{|c|}{ Statistics for each study } \\
\hline $\mathrm{RCT}$ & GA & CS & OR & $\begin{array}{l}\text { Lower } \\
\text { limit }\end{array}$ & $\begin{array}{c}\text { Upper } \\
\text { limit }\end{array}$ & Z-value & P-value \\
\hline Schönenberger et al. ${ }^{5}$ (2016) & $65 / 73$ & $62 / 77$ & 1.966 & 0.779 & 4.962 & 1.431 & 0.153 \\
\hline Berkhemer et al. ${ }^{19}$ (2016) & $41 / 79$ & $86 / 137$ & 0.640 & 0.365 & 1.121 & -1.560 & 0.119 \\
\hline \multirow[t]{2}{*}{ Löwhagen Hendén et al. ${ }^{2}$ (2017) } & $41 / 45$ & $40 / 45$ & 1.281 & 0.321 & 5.119 & 0.351 & 0.726 \\
\hline & & & 1.067 & 0.491 & 2.319 & 1.065 & 0.869 \\
\hline
\end{tabular}

Heterogeneity: $X^{2}=4.405, d f=2(P=0.111) ; I^{2}=54.595 \%$

Study name
Non-RCT
Jumaa et al. ${ }^{28}$ (2010)
Sugg et al. ${ }^{3}(2010)$
Abou-Chebl et al. ${ }^{4}$ (2014)
John et al. ${ }^{23}$ (2014)
Whalin et al. ${ }^{24}$ (2014)
van den Berg et al. ${ }^{22}$ (2015)
Mundiyanapurath et al. ${ }^{21}$ (2015)
Janssen et al. ${ }^{20}$ (2016)

\begin{tabular}{cccccccc}
\multicolumn{2}{c}{ Events/total } & \multicolumn{5}{c}{ Statistics for each study } \\
GA & CS & OR & $\begin{array}{c}\text { Lower } \\
\text { limit }\end{array}$ & $\begin{array}{c}\text { Upper } \\
\text { limit }\end{array}$ & Z-value & P-value \\
$37 / 53$ & $60 / 73$ & 0.501 & 0.217 & 1.159 & -1.615 & 0.106 \\
$6 / 9$ & $43 / 57$ & 0.651 & 0.144 & 2.925 & -0.556 & 0.578 \\
$144 / 196$ & $62 / 85$ & 1.027 & 0.579 & 1.824 & 0.092 & 0.927 \\
$52 / 91$ & $47 / 99$ & 1.475 & 0.832 & 2.616 & 1.331 & 0.183 \\
$96 / 133$ & $70 / 83$ & 0.482 & 0.239 & 0.973 & -2.036 & 0.042 \\
$34 / 70$ & $113 / 278$ & 1.379 & 0.815 & 2.334 & 1.197 & 0.231 \\
$20 / 29$ & $8 / 15$ & 1.944 & 0.539 & 7.019 & 1.015 & 0.310 \\
$43 / 53$ & $25 / 31$ & 1.032 & 0.335 & 3.181 & 0.055 & 0.956 \\
& & 1.014 & 0.783 & 1.313 & 0.106 & 0.916
\end{tabular}

0.01
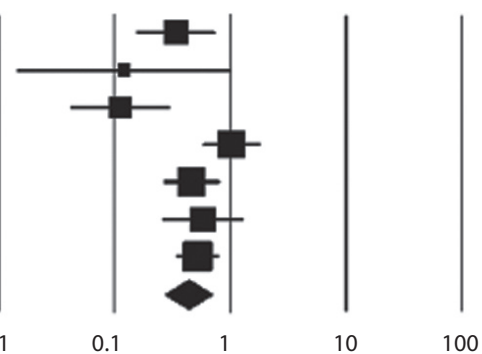

0.0

$1>1$

A

Heterogeneity: $X^{2}=11.295, d f=7(P=0.126) ; I^{2}=38.026 \%$

\begin{tabular}{lrrrrrr}
\hline Total & 0.985 & 0.787 & 1.233 & -0.129 & 0.898 & $\mid$
\end{tabular}

B

Fig. 2. Comparisons of endovascular treatment outcomes according to anesthetic type: general anesthesia (GA) vs. continuous sedation (CS) regarding good functional 3-month outcome (A) and successful recanalization (B) in randomized controlled trials (RCT) or non-RCTs and all studies. OR, odds ratio; $\mathrm{Cl}$, confidence interval. 
Table 2. Meta-analysis of secondary outcomes of mortality, S-ICH and pneumonia

\begin{tabular}{lccccc}
\hline & No. of studies & OR GS vs. CS & $95 \%$ Cl & P-value & $1^{2}$ \\
\hline Mortality on 3 months & 8 & 1.532 & $1.187-1.976$ & 0.001 & 37.948 \\
S-ICH & 7 & 1.125 & $0.767-1.652$ & 0.546 & 0 \\
Pneumonia & 7 & 1.613 & $1.172-2.221$ & 0.003 & 13.028 \\
\hline
\end{tabular}

S-ICH, symptomatic intracranial hemorrhage; OR, odds ratio, GS, general anesthesia; CS, conscious sedation; Cl, confidence interval.
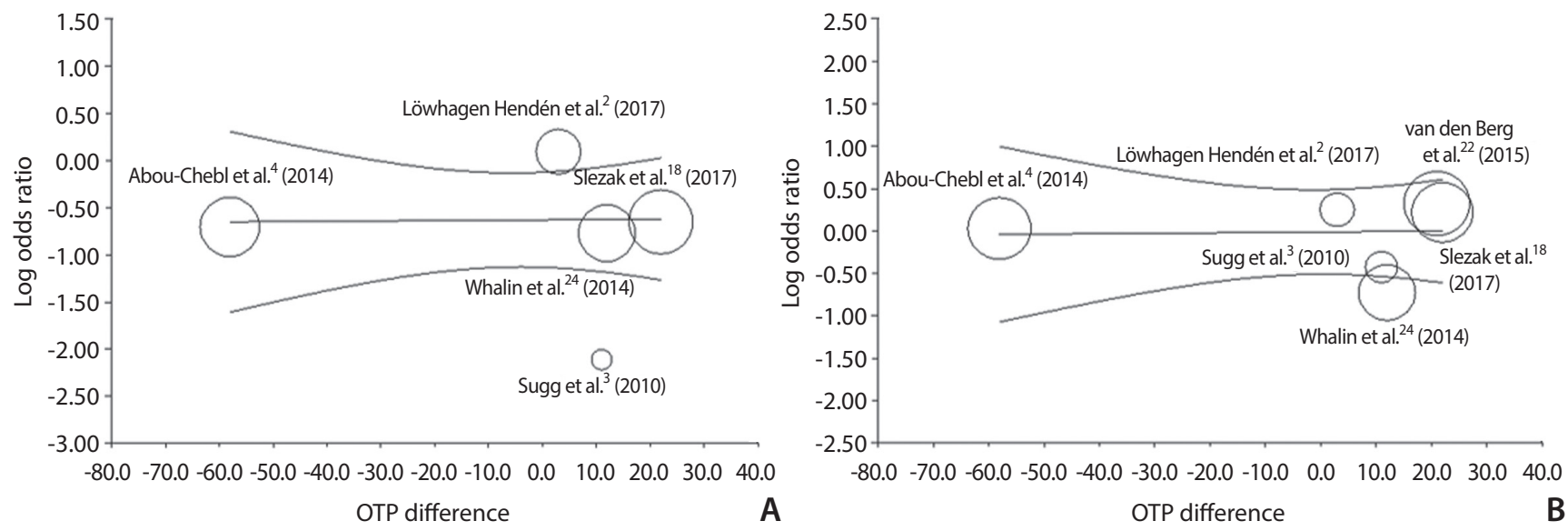

Fig. 3. Meta-regression of differences in onset to groin puncture time (OTP, also descried as the time to treatment) and log of odds ratio of good 3-month outcome $(\mathbf{A})$ and successful recanalization (B) in studies included in this meta-analysis. The difference in the mean or median time OTP was used in five or six studies. Each study is represented by a circle, whose size is proportional to that study's weight in the meta-analysis. The straight line represents the best line of correlation ( $P=0.95$ in $\mathbf{A}$ and $P=0.94$ in $\mathbf{B}$ ).

largely dependent on physicians' experience and intuition. Procedural safety and maintenance of adequate cerebral perfusion in vulnerable ischemic penumbra area are main arguments for choosing anesthesia. ${ }^{29}$ Procedural safety can be assessed through the presence of arterial dissection and $\mathrm{ICH}$ by wire perforation. Although proponent of GA insist that GA has better safety due to immobilization and tight hemodynamic control, ${ }^{29}$ the occurrence of carotid dissection and vessel perforation reportedly does not differ significantly according to anesthesia types. ${ }^{28}$ In our meta-analysis, there was no significant difference in S-ICH occurrence after EVT between the two groups. Hemodynamic instability such as blood pressure (BP) fluctuation and hypotension, during induction or recovery phase, may aggravate ischemic brain injury. ${ }^{6}$ Cerebral vasodilatation by inhalational anesthetic agents could lead to ischemic steal phenomenon, especially in patients with poor collateral. Brinjikji et al. ${ }^{6}$ suggested that poor autoregulation and intraprocedural hypotension in GA can be associated with lower recanalization rates compared to CS. However, during the procedure, fall in intra-operative BP from baseline ( $\mathrm{P}=0.57)$ or anesthesia related complications $(P=0.68)$ did not differ significantly between two an- esthesia types. ${ }^{2}$ Although pre-intubation hypotension can increase cerebral infarct volume, expert anesthesiologist who kept systolic BP suitable for maintaining good collateral circulation through continuous intra-arterial monitoring can help to preserve penumbra area during induction. ${ }^{30}$ Maintenance of normocapnia during the anesthetic procedure is also important because hypocapnic cerebral vasoconstriction during the prolonged and profound ventilation deteriorate the tissue perfusion in ischemic brain lesions. During EVT, CS patents are more vulnerable to sympathetic stimulation compared to GA patients, which could lead to improper ventilation. Accordingly, we should take into account the quality of CS as well as the harmfulness of GA for evaluating EVT outcomes. In clinical circumstances, CS is usually performed by the participating interventionists or neurologists. John et al. ${ }^{23}$ compared EVT outcomes between general and local anesthesia, termed monitor anesthesia care (MAC) by the anesthesia team. The time spent in anesthesia and recanalization was shorter in the MAC group than GA $(92.3 \pm 43.0$ vs. $110 \pm 57.2 ; P=0.045)$ with lower mortality (13.3\% vs. $25.8 \%$; $P=0.04)$. However, parenchymal hemorrhage was more frequently observed in patients receiving GA (26.3\% vs. $10.1 \%$ in 
$M A C ; P=0.003)$ without significant difference in intra-procedural hemodynamic parameter changes. In addition, conversion rate from CS to GA during the procedure was 1\% (1 out of 99). Jumaa et al..$^{28}$ and van den Berg et al. ${ }^{22}$ reported two of 73 subjects (2.7\%) and 10 of 278 subjects (3.7\%) converted from CS to GA, which was accompanied by the tracheal intubation due to agitation and decreased level of consciousness. Therefore, MAC by the expertise of the anesthesiologist could be related to clinical outcomes. More, comparative studies are required that address GA and MAC, or CS delivered by non-anesthesia physicians and MAC delivered by expert anesthesiologists.

It is believed that GA delays the procedure starting time, which can attribute to poor outcomes and increased mortality due to the high respiratory complication rates. Sugg et al. ${ }^{3}$ and Li et al. ${ }^{25}$ reported delayed OTP in GA. In contrast, delayed OTP was observed in ischemic stroke patients who received $C S .{ }^{4}$ Presently, we explored the effect size and explanatory variable of OTP differences according to anesthesia type. For good outcomes on 3-month follow-up, a coefficient of 0.0004 was observed. Regarding successful recanalization, the regression coefficient was 0.0005 ( $P=0.94)$, which indicates no significant linear association between OTP differences and log ORs of successful recanalization. The timing of extubation after EVT with GA is contentious. The occurrence rate of ventilator associated pneumonia is reportedly up to $27 \%$. The risk of ventilator associated pneumonia is highest during the first 5 days. In daily practice, physicians can be reluctant to perform extubation immediately after the procedure, because a number of EVT are done after a day's work. ${ }^{29}$ Whalin et al. ${ }^{24}$ reported that aspiration pneumonia was significantly increased in patients receiving $G A$ ( $n=15,11.2 \%$ vs. $n=3,3.6 \%$ in dexmedetomidine [DEX]). Although GA increased the risk of pneumonia in our meta-analysis, in-hospital morality (OR, 4.4; 95\% Cl, 1.6-12.5) remains significantly associated with $G A$ after adjusting for pneumonia events. ${ }^{26}$ In addition, CS did not improve clinical outcome in multivariate analysis (OR, 1.48; $95 \% \mathrm{Cl}, 0.70-3.12) .^{24}$ Melsen et al. ${ }^{31}$ reported that ventilator associated pneumonia did not increase the mortality in patient with trauma or acute respiratory distress syndrome. More detailed studies are necessary to determine the attributable mortality of pneumonia in ischemic stroke patients with GA.

A meta-analysis done by Brinjikji et al., ${ }^{32}$ reported the significant association of GA with lower good outcome (OR, 0.59; 95\% Cl, 0.29-0.94) after adjusting for baseline NIHSS scale. However, no difference of functional good outcome according to anesthesia type in studies of the stent-retriever/ aspiration era (OR, 0.84; 95\% Cl, 0.67-1.06) was observed. We performed further analysis of clinical and angiographic outcomes in patients who received mechanical thrombectomy only using stent retriever or aspiration catheter. GA significantly decreased good clinical outcome (OR, 0.697; 95\% Cl, 0.549-0.884), but no significant difference was noted in RCTs $(\mathrm{OR}, 1.101 ; 95 \% \mathrm{Cl}, 0.395-3.071)$. Regarding successful recanalization, anesthesia type did not affect outcomes in acute stroke patients who underwent mechanical thrombectomy only (OR, 0.831; 95\% Cl, 0.616-1.122). Li et al..$^{33}$ investigated anesthesia effect on clinical outcomes in patients with anterior circulation stroke and reported inconsistent results. CS was related to improved outcome. However, only for RCTs, GA was associated with improved functional outcomes. ${ }^{33}$ In our initial analysis, GA showed lower good outcomes (OR, 0.564; 95\% Cl, 0.354-0.899). But, the funnel plot was slightly asymmetric, indicating possible publication bias. Using the Trim and Fill method, we imputed two studies and then-recomputed the pooled effect. The adjusted OR suggesting was 0.696 (95\% Cl, 0.424-1.142), suggesting insignificant association between anesthesia types and 3-month outcomes. We think that differences in collateral status and inclusion criteria may lead to conflicting results. Good collateral was associated with higher successful recanalization rates and smaller infarct volume. Nevertheless, collateral degree was not considered for the meta-analyses including ours. For this meta-analysis, we included anterior and posterior circulation stroke patients. Contrary to anterior circulation stroke, clinical efficacy of EVT for posterior circulation stroke has not been demonstrated in RCTs. Weber et al. ${ }^{34}$ reported that EVT in patients with posterior circulation stroke had similar rates of functional outcome and successful recanalization compared to those with anterior circulation stroke. Nevertheless, small number of enrolled patients could be concern to the interpretation. Therefore, additional meta-analyses of individual patients' data dealing with collateral status and thrombus location are warranted.

There are some limitations in this study. First, most studies are retrospective investigations. Accordingly, inherent selection bias such as differences in NIHSS score, proportion of posterior circulation stroke or intravenous recombinant tissue-type plasminogen activator use could have biased the results. Second, the technical diversity of EVT (mechanical thrombectomy with stent retriever or suction aspiration or 
intra-arterial thrombolysis only) and different hemodynamic profile of anesthetic agents, in particular for CS, could attribute to treatment outcomes. John et al. ${ }^{35}$ compared clinical outcomes after EVT between DEX and propofol (PROP). In their study, difference in good 1-month outcome (18.8\% in DEX vs. $22.2 \%$ in $P R O P ; P=0.742$ ) and in-hospital mortality (17.7\% in DEX vs. $8.1 \%$ in PROP; $P=0.225)$ did not reach statistical significance. However, hemodynamic instability and patients needing vasopressor administration were observed frequently in patients with DEX compared to those with PROP. Accordingly, randomized controlled studies including more detailed data at the multiple institutions, including anesthetic agents and stroke severity, are necessary.

\section{CONCLUSION}

In conclusion, GA seemed to decrease good outcome after EVT. However, its efficacy was not demonstrated in RCTs. Successful recanalization did not differ significantly according to anesthesia type. Accordingly, analyses using individual patient data of RCTs are necessary further to elucidate anesthesia effect on procedural and clinical outcomes.

\section{SUPPLEMENTARY MATERIALS}

Supplementary materials related to this article can be found online at https://doi.org/10.5469/neuroint.2019.00045.

\section{Acknowledgments}

This study was supported by a grant from Korean Society of Interventional Neuroradiology.

\section{REFERENCES}

1. Goyal M, Menon BK, van Zwam WH, Dippel DW, Mitchell PJ, Demchuk AM, et al. Endovascular thrombectomy after large-vessel ischaemic stroke: a meta-analysis of individual patient data from five randomised trials. Lancet 2016;387:17231731

2. Löwhagen Hendén P, Rentzos A, Karlsson JE, Rosengren L, Leiram B, Sundeman $H$, et al. General anesthesia versus conscious sedation for endovascular treatment of acute ischemic stroke: the AnStroke trial (anesthesia during stroke). Stroke
2017:48:1601-1607

3. Sugg RM, Jackson AS, Holloway W, Martin CO, Akhtar N, Rymer M. Is mechanical embolectomy performed in nonanesthetized patients effective? AJNR Am J Neuroradio/ 2010;31:1533-1535

4. Abou-Chebl A, Zaidat OO, Castonguay AC, Gupta R, Sun CH, Martin CO, et al. North American SOLITAIRE stent-retriever acute stroke registry: choice of anesthesia and outcomes. Stroke 2014;45:1396-1401

5. Schönenberger S, Uhlmann L, Hacke W, Schieber S, Mundiyanapurath S, Purrucker JC, et al. Effect of conscious sedation vs general anesthesia on early neurological improvement among patients with ischemic stroke undergoing endovascular thrombectomy: a randomized clinical trial. JAMA 2016;316:1986-1996

6. Brinjikji W, Murad MH, Rabinstein AA, Cloft HJ, Lanzino G, Kallmes DF. Conscious sedation versus general anesthesia during endovascular acute ischemic stroke treatment: a systematic review and meta-analysis. AJNR Am J Neuroradiol 2015;36:525-529

7. Rodrigues FB, Neves JB, Caldeira D, Ferro JM, Ferreira JJ, Costa J. Endovascular treatment versus medical care alone for ischaemic stroke: systematic review and meta-analysis. BMJ 2016;353:11754

8. Jeon JP, Kim JE, Cho WS, Bang JS, Son YJ, Oh CW. Meta-analysis of the surgical outcomes of symptomatic moyamoya disease in adults. J Neurosurg 2018;128:793-799

9. Abou-Chebl A, Yeatts SD, Yan B, Cockroft K, Goyal M, Jovin T, et al. Impact of general anesthesia on safety and outcomes in the endovascular arm of interventional management of stroke (IMS) III trial. Stroke 2015;46:2142-2148

10. Nichols C, Carrozzella J, Yeatts S, Tomsick T, Broderick J, Khatri P. Is periprocedural sedation during acute stroke therapy associated with poorer functional outcomes? J Neurointerv Surg 2010;2:67-70

11. Langner S, Khaw AV, Fretwurst T, Angermaier A, Hosten $N$, Kirsch M. Endovascular treatment of acute ischemic stroke under conscious sedation compared to general anesthesia safety, feasibility and clinical and radiological outcome. Rofo 2013;185:320-327

12. Almekhlafi MA, Menon BK, Freiheit EA, Demchuk AM, Goyal M. A meta-analysis of observational intra-arterial stroke therapy studies using the merci device, penumbra system, and retrievable stents. AJNR Am J Neuroradio/ 2013;34:140-145

13. Lapergue B, Blanc R, Guedin P, Decroix JP, Labreuche J, Preda $C$, et al. A direct aspiration, first pass technique (ADAPT) versus stent retrievers for acute stroke therapy: an observational comparative study. AJNR Am J Neuroradiol 2016;37:1860-1865 
14. Kim CH, Jeon JP, Kim SE, Choi HJ, Cho YJ. Endovascular treatment with intravenous thrombolysis versus endovascular treatment alone for acute anterior circulation stroke : a meta-analysis of observational studies. J Korean Neurosurg Soc 2018;61:467473

15. Cho YD, Kim SE, Lim JW, Choi HJ, Cho YJ, Jeon JP. Protected versus unprotected carotid artery stenting : meta-analysis of the current literature. J Korean Neurosurg Soc 2018;61:458-466

16. Kim SE, Kim BJ, Cho SS, Kim HC, Jeon JP. The incidence of hydrocephalus and shunting in patients with angiogram-negative subarachnoid hemorrhage: an updated meta-analysis. World Neurosurg 2018;119:e216-e227

17. Just C, Rizek P, Tryphonopoulos P, Pelz D, Arango M. Outcomes of general anesthesia and conscious sedation in endovascular treatment for stroke. Can J Neurol Sci 2016;43:655-658

18. Slezak A, Kurmann R, Oppliger L, Broeg-Morvay A, Gralla J, Schroth $\mathrm{G}$, et al. Impact of anesthesia on the outcome of acute ischemic stroke after endovascular treatment with the solitaire stent retriever. AJNR Am J Neuroradiol 2017;38:1362-1367

19. Berkhemer OA, van den Berg LA, Fransen PS, Beumer D, Yoo AJ, Lingsma HF, et al. The effect of anesthetic management during intra-arterial therapy for acute stroke in MR CLEAN. Neurology 2016:87:656-664

20. Janssen H, Buchholz G, Killer M, Ertl L, Bruckmann H, Lutz J. General anesthesia versus conscious sedation in acute stroke treatment: the importance of head immobilization. Cardiovasc Intervent Radio/ 2016;39:1239-1244

21. Mundiyanapurath S, Schonenberger S, Rosales ML, Carrilho Romeiro AM, Möhlenbruch M, Bendszus M, et al. Circulatory and respiratory parameters during acute endovascular stroke therapy in conscious sedation or general anesthesia. J Stroke Cerebrovasc Dis 2015;24:1244-1249

22. van den Berg LA, Koelman DL, Berkhemer $O A$, Rozeman $A D$, Fransen PS, Beumer D, et al. Type of anesthesia and differences in clinical outcome after intra-arterial treatment for ischemic stroke. Stroke 2015;46:1257-1262

23. John S, Thebo U, Gomes J, Saqqur M, Farag E, Xu J, et al. Intra-arterial therapy for acute ischemic stroke under general anesthesia versus monitored anesthesia care. Cerebrovasc Dis 2014;38:262-267

24. Whalin MK, Lopian S, Wyatt K, Sun CH, Nogueira RG, Glenn BA, et al. Dexmedetomidine: a safe alternative to general anesthesia for endovascular stroke treatment. J Neurointerv Surg 2014;6:270-275
25. Li F, Deshaies EM, Singla A, Villwock MR, Melnyk V, Gorji R, et al. Impact of anesthesia on mortality during endovascular clot removal for acute ischemic stroke. J Neurosurg Anesthesiol 2014;26:286-290

26. Hassan AE, Chaudhry SA, Zacharatos $H$, Khatri R, Akbar U, Suri $M F$, et al. Increased rate of aspiration pneumonia and poor discharge outcome among acute ischemic stroke patients following intubation for endovascular treatment. Neurocrit Care 2012;16:246-250

27. Davis MJ, Menon BK, Baghirzada LB, Campos-Herrera CR, Goyal M, Hill MD, et al. Anesthetic management and outcome in patients during endovascular therapy for acute stroke. Anesthesiology 2012;116:396-405

28. Jumaa MA, Zhang F, Ruiz-Ares G, Gelzinis T, Malik AM, Aleu $A$, et al. Comparison of safety and clinical and radiographic outcomes in endovascular acute stroke therapy for proximal middle cerebral artery occlusion with intubation and general anesthesia versus the nonintubated state. Stroke 2010;41:11801184

29. Gupta R. Local is better than general anesthesia during endovascular acute stroke interventions. Stroke 2010;41:2718-2719

30. Brekenfeld C, Mattle HP, Schroth G. General is better than local anesthesia during endovascular procedures. Stroke 2010;41:2716-2717

31. Melsen WG, Rovers MM, Bonten MJ. Ventilator-associated pneumonia and mortality: a systematic review of observational studies. Crit Care Med 2009;37:2709-2718

32. Brinjikji W, Pasternak J, Murad MH, Cloft HJ, Welch TL, Kallmes DF, et al. Anesthesia-related outcomes for endovascular stroke revascularization: a systematic review and meta-analysis. Stroke 2017;48:2784-2791

33. Li X, Hu Z, Li Q, Guo Y, Xu S, Wang W, et al. Anesthesia for endovascular treatment in anterior circulation stroke: a systematic review and meta-analysis. Brain Behav 2019;9:e01178

34. Weber R, Minnerup J, Nordmeyer H, Eyding J, Krogias C, Hadisurya J, et al. Thrombectomy in posterior circulation stroke: Differences in procedures and outcome compared to anterior circulation stroke in the prospective multicentre REVASK registry. Eur J Neurol 2019;26:299-305

35. John S, Somal J, Thebo U, Hussain MS, Farag E, Dupler S, et al. Safety and hemodynamic profile of propofol and dexmedetomidine anesthesia during intra-arterial acute stroke therapy. J Stroke Cerebrovasc Dis 2015;24:2397-2403 